\title{
The technique of cutting open the bronchus during VATS left upper lobectomy with complicated hilar anatomy
}

\author{
Feng Lin ${ }^{1,2}$, Shiyou Wei ${ }^{1}$, Chengwu Liu ${ }^{1,2}$, Qiang $\mathrm{Pu}^{1,2}$, Jiandong Mei ${ }^{1,2}, \mathrm{Hu} \mathrm{Liao}^{1,2}$, Lunxu Liu ${ }^{1,2}$ \\ ${ }^{1}$ Department of Thoracic Surgery, West China Hospital, Sichuan University, Chengdu 610041, China; ${ }^{2}$ Western China Collaborative Innovation \\ Center for Early Diagnosis and Multidisciplinary Therapy of Lung Cancer, Chengdu 610041, China \\ Correspondence to: Lunxu Liu. Department of Thoracic Surgery, West China Hospital, Sichuan University, No. 37, Guoxue Alley, Chengdu 610041, \\ China. Email: lunxu_liu@aliyun.com.
}

Submitted Oct 10, 2018. Accepted for publication Oct 11, 2018.

doi: $10.21037 /$ jtd.2018.10.119

View this article at: http://dx.doi.org/10.21037/jtd.2018.10.119

\section{Introduction}

Video-assisted thoracic surgery (VATS) lobectomy has been the most common procedure for both malignant and benign pulmonary diseases $(1,2)$. However, complicated hilar anatomies, such as tumor invasion, lymphadenopathy, calcification and dense inflammatory adhesion, still pose significant challenges for this type of surgery, and most vascular injuries and conversions can be attributed to these complicated hilar anatomies. In reports from the relevant literature, the successful rate of management of accidental vascular injury under thoracoscopy was observed only to range from 0 to $26.9 \%(1,2)$, and the incidence of intraoperative conversion was, for various reasons, found to be as high as $23 \%(3,4)$. Various techniques have been used to troubleshoot complicated hilar situations and subsequently reduce the incidence of massive bleeding or conversion to thoracotomy (5-9). Here we describe a technique of cutting open the bronchus for the management of unexpected severe hilar adhesion (Figure 1).

\section{Operative techniques}

This was a planned VATS left upper lobectomy performed under general anesthesia with double-lumen intubation. The patient was placed in the right lateral decubitus position. A 1.5 -cm observation port for the thoracoscope was made in the seventh intercostal space at the midaxillary line. A $3-\mathrm{cm}$ main utility incision and $2-\mathrm{cm}$ assistant utility incision were made at the anterior axillary line in the third intercostal space and at the posterior axillary line in the ninth intercostal space respectively.

Following the strategy of "single direction", we transected the superior pulmonary vein first. However, when we tried to manage the left upper bronchus, severe adhesions between the bronchus and the pulmonary artery
(PA) were encountered. The endo-stapler could not pass through the interspace between the bronchus and the PA. Therefore, we cut open the bronchus with endoscopic scissors before managing the arterial branches and created an easier access for dissecting posterior arterial branches from the anterior approach. After that, the dense adhesion between the PA and the bronchus was identified. Sharp dissection was performed with scissors and an electrocautery hook. We then sequentially ligated and cut off the lingual artery and the posterior artery branches of the PA. However, the apical anterior arterial branch of the PA could not be dissected due to dense inflammatory adhesions. In order to avoid catastrophic bleeding or accidental damage to the PA, the technique of prophylactically clamping the PA using detachable endoscopic bulldog clamps was also applied. When sufficient length was separated out, the anterior artery branch and partial trunk of the PA was resected, and angioplasty was simultaneously performed using the endo-stapler. At last, the bronchial stump was closed with the endo-stapler.

\section{Comments}

Complicated hilar anatomies have consistently proven to be a technical challenge for surgeons, as they commonly lead to conversions from VATS to thoracotomy, and even catastrophic intraoperative complications during VATS lobectomy. In order to cope with these complex situations, some techniques have been designed $(8,9)$. Using our collection of experience as a resource, we gradually developed a series of strategies aimed at managing different types of complicated hilar anatomies.

Commonly, the bronchus is transected and its stump is simultaneously closed with an endo-stapler in VATS lobectomy. However, there may occasionally be adhesions 


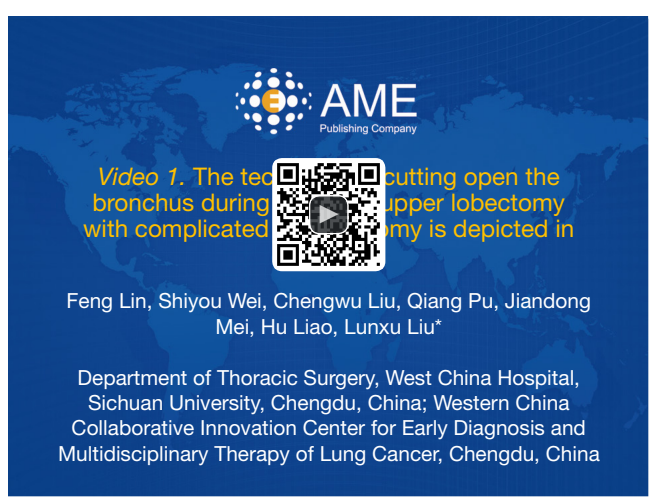

Figure 1 The technique of cutting open the bronchus during VATS left upper lobectomy with complicated hilar anatomy is depicted in this video (10). VATS, video-assisted thoracic surgery. Available online: http://www.asvide.com/article/view/28639

or lymphadenopathy appearing just between the target artery and the bronchus. Because of this, the artery or bronchus can only be partially dissected while the inner most part cannot be dissected by either anterior or posterior approach. Under such circumstances, it is not possible for the endo-stapler to pass through the interspace between the bronchus and the PA. Therefore, in such cases, we choose to cut open the bronchus with scissors to carve out a path to dissect the posterior arterial branches from the anterior approach. This method has been mainly used during left upper lobectomy. In addition, if we encounter a completely frozen hilar caused by different non-neoplastic lesions, such as carbonized peri-arterial/peri-bronchial lymphadenopathy, the strategy of "firing the bronchus and PA together" is employed (11). In these strategies, the technique of prophylactically clamping the PA plays an important role as it ensures the safety of the operation (12).

To conclude, when encountering any emergency during VATS lobectomy, calmness and effective technical strategy are indispensable.

\section{Acknowledgements}

None.

\section{Footnote}

Conflicts of Interest: The authors have no conflicts of interest to declare.

Informed Consent: Written informed consent was obtained from the patient for publication of this manuscript and any accompanying images.

\section{References}

1. Yamashita S, Tokuishi K, Moroga T, et al. Totally thoracoscopic surgery and troubleshooting for bleeding in non-small cell lung cancer. Ann Thorac Surg 2013;95:994-9.

2. Dunning J, Walker WS. Pulmonary artery bleeding caused during VATS lobectomy. Ann Cardiothorac Surg 2012;1:109-10.

3. Hanna JM, Berry MF, D'Amico TA. Contraindications of videoassisted thoracoscopic surgical lobectomy and determinants of conversion to open. J Thorac Dis 2013;5:S182-9.

4. Berry MF. Pulmonary Artery Bleeding During VideoAssisted Thoracoscopic Surgery: Intraoperative Bleeding and Control. Thorac Surg Clin 2015;25:239-47.

5. Jiang L, Wu L, Roque SR, et al. A novel tourniquet technique for transient pulmonary artery occlusion during video-assisted thoracoscopic surgery. J Thorac Cardiovasc Surg 2018;156:816-8.

6. Zhang Z, Huang J, Yin R, et al. A new technique for partial removal of the pulmonary artery in video-assisted thoracic surgical lobectomy. J Thorac Cardiovasc Surg 2012;144:512-4.

7. Kamiyoshihara M, Nagashima T, Ibe T, et al. A tip for controlling the main pulmonary artery during videoassisted thoracic major pulmonary resection: the outsidefield vascular clamping technique. Interact Cardiovasc Thorac Surg 2010;11:693-5.

8. Nakanishi R, Oka S, Odate S. Video-assisted thoracic surgery major pulmonary resection requiring control of the main pulmonary artery. Interact Cardiovasc Thorac Surg 2009;9:618-22.

9. Watanabe A, Koyanagi T, Nakashima S, et al. How to clamp the main pulmonary artery during video-assisted thoracoscopic surgery lobectomy. Eur J Cardiothorac Surg 2007;31:129-31.

10. Lin F, Wei S, Liu C, et al. The technique of cutting open the bronchus during VATS left upper lobectomy with complicated hilar anatomy is depicted in this video. Asvide 2018;5:877. Available online: http://www.asvide.com/article/view/28639

11. Liu C, Ma L, Pu Q, et al. How to deal with benign hilar or interlobar lymphadenopathy during video-assisted thoracoscopic surgery lobectomy-firing the bronchus and pulmonary artery together. J Vis Surg 2016;2:26.

12. Ma L, Mei J, Liu C, et al. Precontrol of the pulmonary artery during thoracoscopic left upper lobectomy and systemic lymph node dissection. J Thorac Dis 2016;8:E317-8.

Cite this article as: Lin F, Wei S, Liu C, Pu Q, Mei J, Liao $\mathrm{H}$, Liu L. The technique of cutting open the bronchus during VATS left upper lobectomy with complicated hilar anatomy. J Thorac Dis 2018;10(11):6269-6270. doi: 10.21037/ jtd.2018.10.119 